\title{
DETERMINATION OF DETAILED MORPHOLOGICAL FEATURES FOR PHENOTYPING OF SUGAR BEET PLANTS USING 3D-STEREOSCOPIC DATA
}

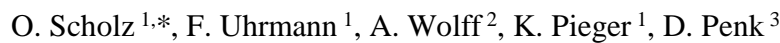 \\ ${ }^{1}$ Fraunhofer Development Center X-ray Technology, 90768 Fürth, Germany \\ - (oliver.scholz, franz.uhrmann, katharina.pieger)@iis.fraunhofer.de \\ ${ }^{2}$ Strube Research GmbH, Hauptstr. 1, 38387 Söllingen, Germany - a.wolff@ strube.net \\ ${ }^{3}$ Friedrich-Alexander University Erlangen-Nuremberg, Computer Graphics Group, 91058 Erlangen, Germany \\ - dominik.penk@fau.de
}

ICWG II/III: Pattern Analysis in Remote Sensing

KEY WORDS: Remote sensing, Phenotyping, Precision Farming, Plant morphology, Plant modelling, Sugar beet, Leaf model, 3D

\begin{abstract}
:
The sugar beet is the primary source of sugar in Europe and large parts of the world. Tools to determine plant traits with high precision and high throughput are required for the breeding process to quantify the effects of genetic and environmental factors on plant development and yield. In this work, we propose a method to gain a limited yet significant set of descriptive parameters for sugar beet plants. Using optical methods, a 3D representation of each plant is generated and subsequently segmented manually. A customized leaf model developed specifically for sugar beet plants then models the leaves, yielding a vector of descriptive parameters for each leaf. The resulting data is then compared to plant assessments of the same plants performed by sugar beet experts in order to evaluate the viability of automatic plant assessment in the sugar beet breeding process.
\end{abstract}

\section{INTRODUCTION}

\subsection{Significance of morphological features for breeding}

The sugar beet (Beta vulgaris L.) is the primary source of sugar in non-tropical regions due to the high percentage of sucrose in its root aimed at sugar refining (Dillen, 2014), contributing 20\% to the world sugar production (UN, 2009). The growing world population's need for food demands robust plants growing in changing climates with optimal yields.

For the breeder this translates to multiple challenges. A higher sugar content of typically $15-20 \%$ of the beet's weight is one of various, sometimes conflicting goals, as it normally decreases tolerance of the plants to stress factors like drought, heat or diseases. The sugar beet plant's DNA carries the genetically encoded behavior of the plant as a reaction to its environment. The resulting morphology of the plant in response to environmental conditions is of great interest to the breeder. Properties like number of leaves, (individual) leaf area(s), plant height, leaf angles, etc. are key to breeding efforts attempting to create more robust plants in combination with higher yields. Changes in a plant's DNA may result in very small, but biologically important changes of the plant parameters, so a precise determination of these parameters is vital. Correlating leaf properties gained using above ground sensing of the plants with sugar content or stress resistance allows the breeder to optimize the breeding process.

However, determining these parameters is time consuming and expensive as plant assessment of sugar beets is currently carried out visually in the field by skilled personnel. In addition to the effort associated with visual plant assessment, there is the problem of inaccuracy inherent in any subjective procedure. An accurate non-destructive actual measurement of said parameters is virtually impossible, let alone in large plant quantities required by the breeding process.

\subsection{D-Phenotyping}

The process of describing a plant's observable physical characteristic (morphology) as a reaction of its genotype and in response to the environment is commonly referred to as "plant phenotyping".

Many traits can be monitored by a phenotyping system (Cendrero, 2017). Various commercial systems have been available estimating parameters like leaf area or plant height from side view and/or top view images for a few years now. However, basing these estimations solely on 2D data introduces significant errors: the plant itself may obscure some parts of the plant, or the leaf area calculations cannot take the bending and leaf angles into consideration. Leaf angles also vary due to variations of water content (turgor) and time of day. The results thus lack the accuracy the breeder desires to detect even minute effects of breeding.

In order to calculate plant parameters as precisely as possible it is unavoidable to capture a 3D-representation of the plant. This approach ensures a sufficient level of detail of the plant and provides a basis for further algorithmic processing.

\subsection{Field phenotyping}

Phenotyping is performed on plants in the greenhouse as well as in the field. Both applications have their advantages, so it is vital for the breeder to be able to not only gather data regarding

\footnotetext{
* Corresponding author
} 
a plant's performance in controlled environments but also in the actual soil and environmental conditions it is bred for. This in turn poses a number of challenges for $3 \mathrm{D}$-acquisition and all subsequent processing tasks.

\subsection{Related work}

Many different groups are working on 3D-modeling of different plants, e.g. Gelard et al. for sunflowers. Often each plant parameter is calculated from the $3 \mathrm{D}$ data using a separate analytical approach, e.g. using an averaged normal and curvature around the points of a leaf to segment leaves and determine a leaf angle (Paulus et al). Also, the term "model" is used for a variety of different concepts.

The method presented in this work is based on (Uhrmann, 2009), using a geometric model capable of generating a 3D representation of a leaf designed to replace the amount of data in a leaf's point cloud to very few mathematical parameters describing the leaf with sufficient accuracy. The parameter vector generated by the model describes the leaf completely with sufficient detail, eliminating the need for separate algorithms for each plant parameter. The reduction of the geometrical complexity of a high-precision 3D scan to a manageable vector of features, yet preserving the essence of information relevant for the analysis of biological are the basic concept of this approach.

\section{3D-PHENOTYPING OF SUGAR BEET PLANTS}

\subsection{Method overview}

We propose a straightforward method to determine a sugar beet's key parameters consisting of this sequence of steps:

1. Optical acquisition of 3D-data (point cloud)

2. Segmentation into single plants/leaves

3. Model-based leaf parameter extraction

4. Parameter assessment

Using non-destructive 3D optical data acquisition must be able to provide data of the plant with the required level of detail while at the same time not affecting the plant adversely. The generated 3D data must then be assigned to individual plants and plant organs (leaves, etc.) respectively. A model-based approach can then be used to efficiently describe each leaf's morphology with a sufficient accuracy preserving the characteristics the breeder is interested in using only relatively few parameters. These leaf parameter vectors can then be merged into a compact dataset mirroring a plant and providing a basis for the assessment of the plant.

The basic sequence of steps is applicable to both greenhouse as well as field use, but details may need to be adapted to the individual situation as required. If both applications are sought, care must be taken with each step to assure equal suitability for both scenarios.

\subsection{Data capture}

Capturing the morphology of a plant with a high level of detail poses unique challenges. While many different 3D-acquisition methods are available, only few are suited for this purpose. Laser-based triangulation methods like the "Sheet-of-Light"method provide excellent accuracy of the resulting point cloud paired with a fair throughput. However, it is best suited for greenhouse applications where the plants are moved to a stationary scanner or a scanner is moved uniformly. Using this method in the field poses multiple challenges, like uniformly moving the measurement sensor over the plants, lighting conditions, wind, etc. Structured-light based methods also suffer from inherent environmental weaknesses like sensitivity to wind, sunlight, etc.

Stereophotogrammetry using two or more cameras on the other hand is very well suited for outdoor applications. During daytime hours, exposure times can be kept low, making the data acquisition process insensitive to wind and robust across a wide range of lighting conditions and providing colour data without additional sensors. Depth resolution and overall data quality is not as detailed as using the triangulation methods though, so it should be considered only for applications involving outdoor plant phenotyping.

\subsection{Plant segmentation}

Segmenting the 3D scan is one of the key challenges of the proposed method. The point cloud consists of a very large number of individual points, each of which is potentially independent of its neighbouring points. Triangulation of point clouds faces similar challenges when deciding which points and triangles should belong together.

Reliable segmentation of the points into categories like plantsoil/other, different plant instances (for scans of multiple plants, usually in the field) and individual plant organs like individual leaves is a prerequisite for subsequent plant modelling. The quality of the plant model parameters is directly dependent on the quality of the 3D-point cloud segmentation.

\subsection{Plant modelling}

The core of the proposed method is a universal leaf model developed by (Uhrmann, 2009). This model is able to create a simple, three dimensional leaf based on a few numerical parameters. Each of the parameters controls a physical property of the generated leaf, like e.g. leaf bending or rolling (see Fig. 1). The entire parameter vector creates an individual leaf.

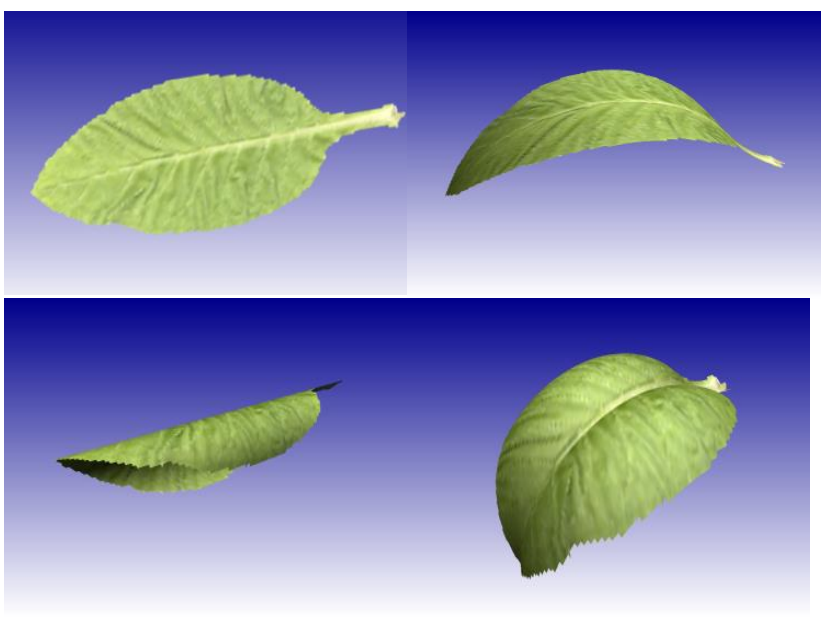

Figure 1. Generic leaf model: bending and rolling 
The idea behind this approach is modify the computer generated model leaf to match the $3 \mathrm{D}$ data points generated by the camera scan. When the match is optimal, the parameters capture the essence of the leaf for further analysis without the need to store or process the $3 \mathrm{D}$ point cloud. Additionally, the majority of the mathematical parameters for the computer generated leaf model bear an immediate meaning for the breeder, simplifying their interpretation for the breeding process.

The core of the process is to create an artificial leaf from an initial leaf parameter vector, and subsequently continue to iteratively minimize the distance between the input point cloud of the real leaf and the generated artificial leaf using non-linear optimization methods. The resulting parameter vector approximates the leaf as best as possible within the bounds of the given leaf model.

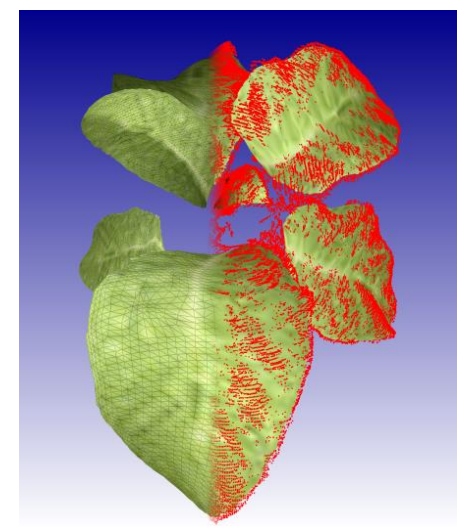

Figure 2. Scanned tobacco plant

Fig. 2 shows the point cloud of laser-scanned tobacco leaves. The approximation is not exact, which is intentional. A more exact approximation requires additional parameters, and the model is designed to use as few parameters as possible to capture only the essential morphology of a plant.

\subsection{Performance assessment}

In order to assess the quality of the model based parameter extraction, it is necessary to compare the data generated by the proposed computerized phenotyping method with plant assessments performed by a human expert on the identical plants used by the algorithm. The breeder will only accept the new method for sugar beet phenotyping if the model based algorithm correlates sufficiently with the visual assessment.

\section{EXPERIMENTS}

\subsection{Greenhouse trials}

In preparation of the greenhouse, trials 64 sugar beet plants were raised in a controlled environment (see Fig. 3). Each plant was potted individually and the pots were placed in seven rows (= genotypes) of about nine plants, offering the possibility to scan all plants of a row in a single scan.

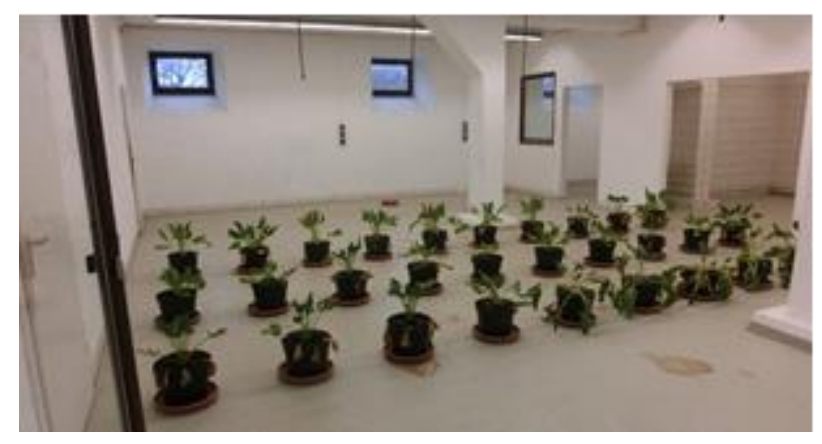

Figure 3. Sugar beet plants in pots

\subsection{Multi-view Stereoscopy}

A multi-view stereoscopic setup was chosen for the experiments since this method is suitable for both greenhouse and field use. For this purpose three JAI AD130 cameras (see Table 1 for technical data) were mounted to a rig (see Fig. 4), with a top view camera and two side view cameras capturing the important downward view of the plant as well as the two side views offering two 3D views at different angles. The goal was to capture the plants as completely as possible using three cameras and yet be able to generate depth information using the three cameras as two camera pairs.

\begin{tabular}{|l|l|}
\hline Camera resolution & $1296 \times 966$ \\
\hline Scan width & $550 \mathrm{~mm}$ \\
\hline Distance between views & $50 \mathrm{~mm}$ \\
\hline $\begin{array}{l}\text { Measurement volume of } \\
\text { single view }\end{array}$ & $400 \mathrm{~cm}^{3}$ \\
\hline Angle of lateral cameras & $44^{\circ}$ \\
\hline
\end{tabular}

Table 1. Technical data of JAI AD130 cameras

A small app was developed specifically for this purpose controlling the three cameras and providing the capability to trigger all three cameras simultaneously with identical settings.

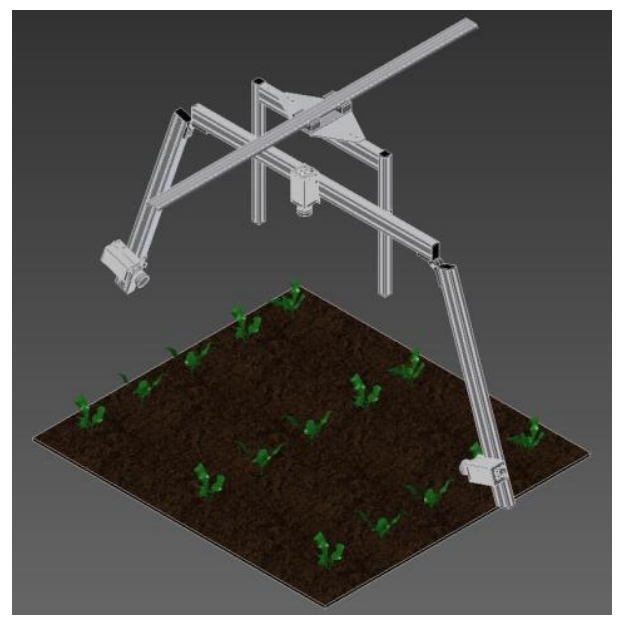

Figure 4. Measurement rig 
The plants were captured at intervals of $5 \mathrm{~cm}$. For each campaign, the camera rig was moved across each row of plants, creating a set of three-color images for each exposure. The commercial "Agisoft Photoscan" software was then used to process the 2D color images and generate color point clouds for a row of plants at a time.

The resulting color point-cloud data for a single pot is shown in Fig. 5. Since the subsequent modelling algorithm is robust regarding spatial resolution, noise, etc. data quality was not a key issue. As long as the 3D images represented a plant well enough for a human assessment, the image quality was considered sufficient. The detail level was analyzed with sugar beet experts and determined to capture all relevant details of the plant, so it was not required to improve or vary the data acquisition setup.

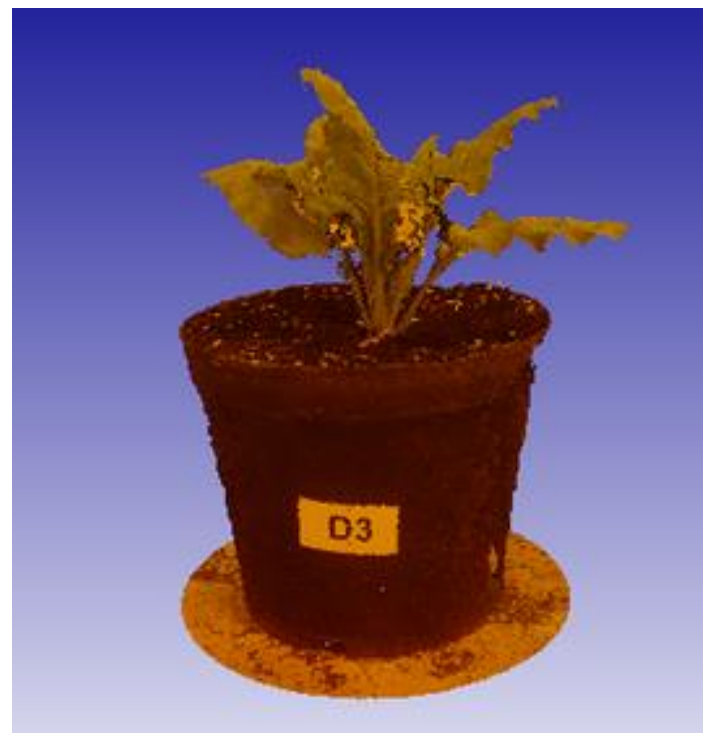

Figure 5. Single sugar beet point cloud

\subsection{Segmentation}

The segmentation of the point cloud into single plants and individual leaves is critical for the subsequent modelling step, so sugar beet experts segmented the 3D data manually into individual plant components. Each set of manually created leaf clouds for a plant was then ready to for processing through the modelling algorithms separately.

\subsection{Sugar beet leaf model}

The existing simple generic leaf model was determined to be insufficient to accurately model some key geometric features important to the sugar beet breeders. In order to model the sugar beet leaf more realistically, more parameters were added to the existing model. Instead of one basic leaf shape, the model would now be based on three basic sugar beet leaf shapes. In addition, the assumption of a convex leaf outline built into the simple leaf model oversimplifies the shape of a real sugar beet leaf sometimes showing a wavy outline. Similarly, the surface of a real sugar beet leaf contains bulges, which were not present in the original leaf model.

Apart from these major improvements resulting in additional leaf parameters, a number of smaller adjustments were required to achieve a sufficient similarity between model leaf and reality. Thin leaf stems were often not or not sufficiently present in the point cloud because of resolution limitations, resulting in incorrect angles. This was compensated by using additional weights along the leaf centre and taking into account the centre of the plant.

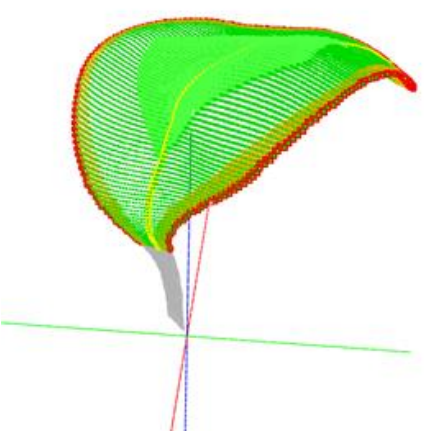

Figure 6. Regionalized leaf view

Segmenting a leaf into separate regions ("Regionalization") was performed to facilitate the calculation of different parameters. Fig. 6 shows different areas of a leaf colour coded for different feature calculations. The stem (grey) for instance was used for the stem angle, the entire leaf (green) for the leaf shape, edge (red) for waviness, wide edge (orange) for bulges, etc.

The model based feature extraction was timed using an Intel Core i7 with 8 codes at $3 \mathrm{GHz}$, resulting in a processing time of 0.6 seconds for each leaf. A sugar beet plant with 8 leaves can thus be evaluated in under 5 seconds.

\subsection{Results and Analysis}

The acceptance of the new phenotyping method by the breeder depends on the correlation of the automatically generated parameters with individual visual assessments of the 64 plants in the experiment performed by sugar beet experts.

For this purpose, a test sample was prepared using plant measurements performed in April/May 2017, consisting of 64 individual leaves of various growth stages and breeds. The goal was to cover as wide a range of phenotypical traits as possible. Three sugar beet experts then visually assessed the leaf traits, and the average of the experts' grades represented the reference for the automatically generated parameters.

Although the model yields more than 20 parameters per leaf, leaf angle and stem angle are not only particularly important parameters for the sugar beet breeder, but their definition also seemed straightforward. Other parameters like e.g. "bulginess" however are not well suited for a comparison of algorithmic and expert assessments since their definition was introduced during this research and the experts are not yet experienced applying them. So leaf and stem angle were selected to analyse the performance of the model based parameter generation.

Figure 7 shows the correlation between manually and algorithmically generated assessments of the leaf angle. 


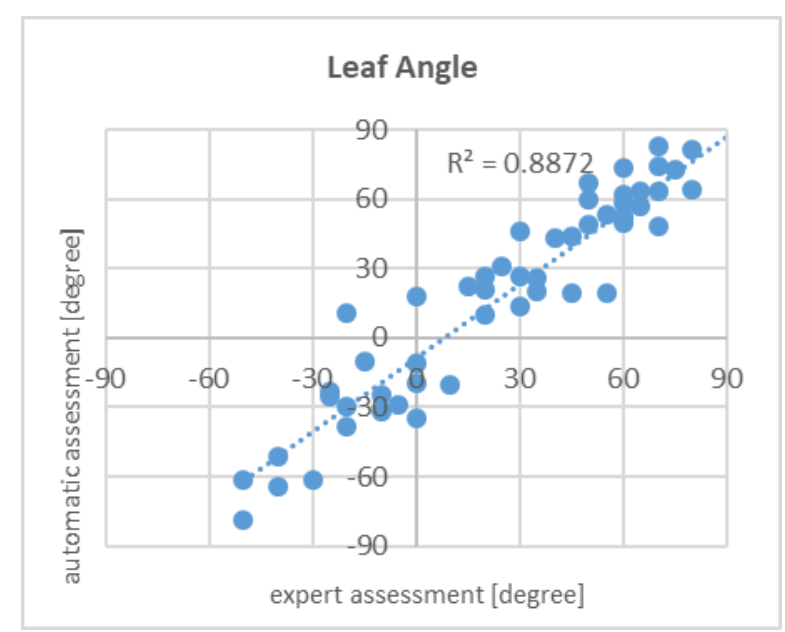

Figure 7. Leaf angle visualization

While there is good correlation between the two assessments, individual values sometimes deviated significantly. The experts estimated the leaf angle in 10-degree steps, so some deviations were to be expected. Yet, some leaves showed considerably higher deviations, so the cause for the deviations was investigated further revealing interesting insights.

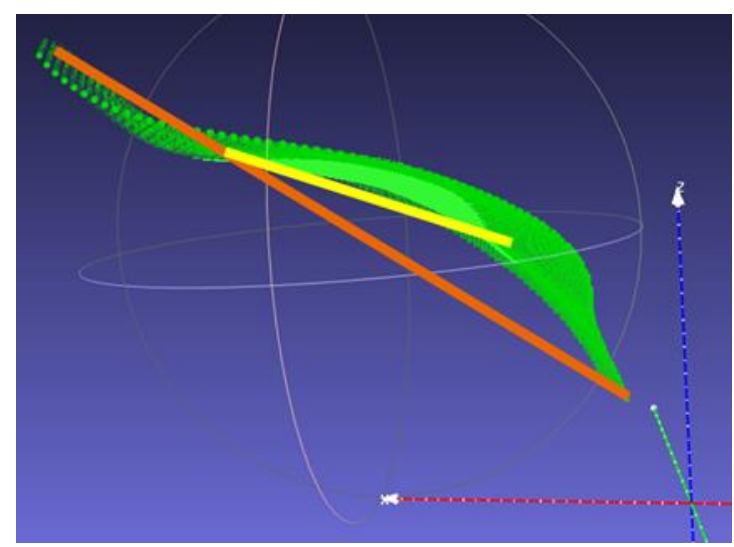

Figure 8. Leaf angle visualization

Figure 8 for example shows a leaf with a considerable deviation between expert and algorithmic assessment. Discussions with the experts showed that the visual assessment was based on the orange line shown, resulting in a leaf angle of 45 degrees. The leaf model however uses the yellow line, resulting in a leaf angle of merely 20 degrees.

Discussions with the experts surprisingly resulted in the understanding that the bend of the leaf only gives the impression of a leaf with a steep angle of 45 degrees. In reality, the major part of the leaf has an angle of 20 degrees so the effective angle of this particular leaf is indeed 20 degrees and the experts agreed that the calculated effective leaf angle is actually correct.

Similar deviations were determined to take place frequently, explaining the correlation results for this particular parameter.

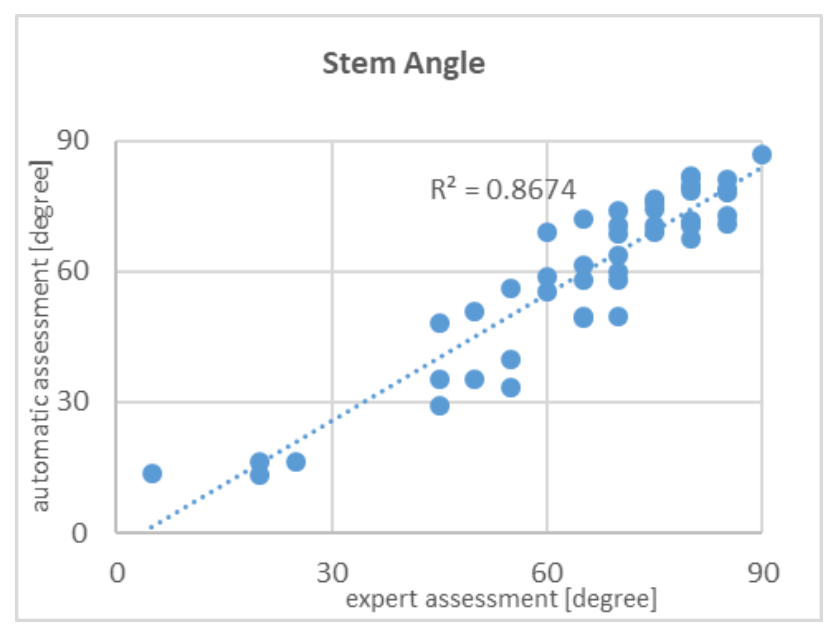

Figure 9. Stem angle visualization

The stem angle (Fig. 9) shows a good overall correlation, but the leaf curvature (Fig. 10) - while showing some correlation also shows some significantly deviating individuals.

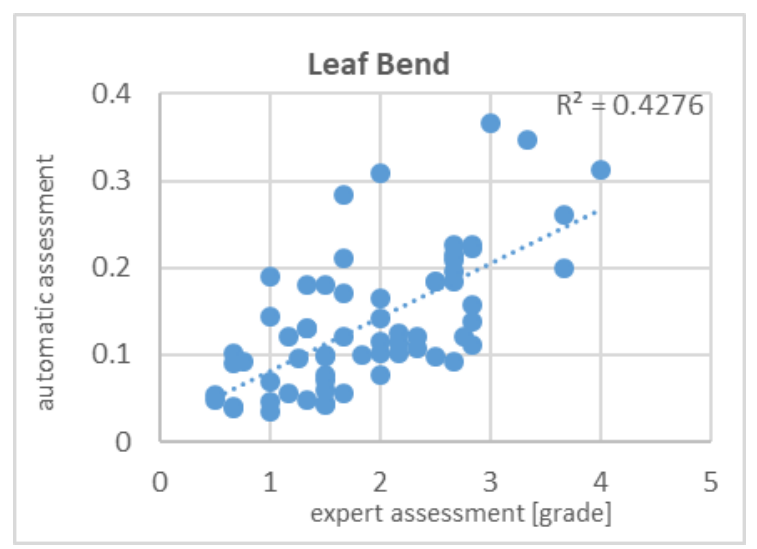

Figure 10. Curvature visualization

The manual curvature assessment was performed on a scale of 0-5 while the model resulted in a parameter range of 0 to 0.4 . Again, the deviations were analysed and three main causes were determined.

Since many leaves have a significant change of angle in the transition from stem to leaf, the determination of this point is critical. Slight differences between manual and algorithmic assessment in the determination of the transition point stem/leaf result in a large difference in leaf curvature.

A second reason lies in a situation not covered in the current leaf model, which would require an additional basic leaf shape implementation.

Finally, the experts' assessments showed significant deviations from each other, sometimes by more than one grade: the average of the standard deviation is 0.7 . Discussions have shown that the curvature trait is much more abstract than other parameters and thus more difficult to assess.

As a result, the analysis of the experiments has proven the hypothesis that a human expert's visual assessment is prone to 
errors while an algorithmic measurement is objective, explainable and reproducible.

\section{FUTURE WORK}

\subsection{Field trials}

The successful application of the algorithmic assessments has encouraged the intention to move the experiments to a field environment. More data will be gathered in the course of 2019 for additional analysis.

\subsection{Data capture}

A commercially available industrial stereoscopic camera natively generating colour point clouds will replace the rig used in this work. The primary reasons for this decision were the effort that would be required to waterproof the setup as well as the time required to process the $2 \mathrm{D}$ images into $3 \mathrm{D}$ using an external software tool. Using the new camera setup will allow the acquisition of 3D-data in the field in real-time. Although the resolution of the new setup will be inferior to the existing setup, it is expected to still be sufficient for the model-based approach. The camera's capability to generate high-speed 3D-data will prove beneficial in windy conditions in the field when leaves move during measurement which causes motion blur.

\subsection{Automatic segmentation}

The manual segmentation of the 3D-data proved to be a significant task in this work. (Brabandere, 2017) has demonstrated a 2D segmentation method, which may be a suitable basis for a segmentation algorithm for the $3 \mathrm{D}$ point clouds of sugar beet leaves.

\subsection{New assessment scheme}

Since the work has revealed weaknesses in the visual plant assessment scheme, the breeder has decided to revise their assessment scheme for the leaf curvature trait and repeat the experiment. The purpose is to reduce the deviation of the experts' assessments, and demonstrate potential for the improvement or the limits of visual assessments through experts.

\section{CONCLUSIONS}

This work has shown that automatic assessment of sugar beets using 3D images and a model based parameter generation is not only possible but has the potential to meet the breeder's expectation of an accurate and thus objective plant assessment tool. The work has also demonstrated the inherent weakness of human assessment as performed today and the inability of the current process to adequately quantify small parameter variations in a plant population.

The application of this method to sugar beet plants in the field possibly using autonomous phenotyping vehicles similar to the one presented in (Thompson, 2018) may become a precision tool helping sugar beet breeders to produce better seeds providing higher yields in the future.

\section{ACKNOWLEDGEMENTS}

The research in this work was part of the "For3D" research collaboration supported by the "Bayerische Forschungsstiftung (BFS)". The authors would like to thank all partners within "For3D" for their cooperation and valuable contributions. The authors are grateful to Strube Research for their invaluable support capturing and segmenting the plant data as well as discussions regarding sugar beet modelling.

\section{REFERENCES}

Behmann, J., Mahlein, A.-K., Paulus, S., Dupuis, D., Kuhlmann, H., Oerke, E.-H., Plümer, L., 2016. Generation and application of hyperspectral 3D plant models: methods and challenges. Machine Vision and Applications(2016), 27, 611624. doi.org/10.1007/s00138-015-0716-8.

Cendrero-Mateo, M. P., Muller, O., Albrecht, H., Burkart, A., Gatzke, S., Janssen, B., Keller, B., Körber, N., Kraska, T., Matsubara, S., Li, J., Müller-Linow, M., Pieruschka, R., Pinto, F., Rischbeck, P., Schickling, A., Steier, A., Watt, M., Schurr, U., Rascher, U., 2017: Field Phenotyping: Challenges and Opportunities. Terrestrial Ecosystem Research Infrastructures, 53-80. doi.org/10.1201/9781315368252-4.

De Brabandere, B., Neven, D., Van Gool, L., 2017. Semantic Instance Segmentation with a Discriminative Loss Function. Deep Learning for Robotic Vision Workshop at CVPR 2017. arXiv:1708.02551.

Dillen, K., Demont, M., 2014. Sugar beet. Handbook on agriculture, biotechnology and development. 661-674. doi.org/10.4337/9780857938350.00048.

Gelard, W., Devy, M., Herbulot, A., Burger, P., 2017: Modelbased Segmentation of 3D Point Clouds for Phenotyping Sunflower Plants, Proceedings of the 12th International Joint Conference on Computer Vision, Imaging and Computer Graphics Theory and Applications (VISIGRAPP 2017). 459467. ISBN: 978-989-758-225-7, doi.org/ $10.5220 / 0006126404590467$.

Paulus, S., Dupuis, J., Mahlein, A.-K., Kuhlmann, H., 2013. Surface feature based classification of plant organs from 3D laserscanned point clouds for plantphenotyping. BMC Bioinformatics 2013. doi.org/10.1186/1471-2105-14-238.

Thompson, A., Thorp, K. R., Conley, M., Andrade-Sanchez, P., Heun, J. T., Dyer, J. M., White, J. W., 2018. Deploying a Proximal Sensing Cart to Identify Drought-Adaptive Traits in Upland Cotton for High-Throughput Phenotyping. Frontiers in Plant Science, 9, 2018. doi.org/10.3389/fpls.2018.00507.

Uhrmann, F., Hügel, C., Paris, S., Scholz, O., Zollhöfer, M., Greiner, G., 2013. A Model-based Approach to Extract Leaf Features from 3D Scans, Proceedings of the $7^{\text {th }}$ International Conference on Functional-Structural Plant Models, June 2013, Saariselkä, Finland.

United Nations, 2009. Agribusiness Handbook: Sugar beet white sugar. Food and Agriculture Organization, United Nations (2009). 